\title{
Analytical challenge on Real Driving Emission
}

Hiroshi Nakamura, HORIBA Ltd.

This manuscript is not available according to publishing restriction. Thank you for your understanding. 